\title{
3D Digitalization of historical cipher machines using computed tomography
}

\author{
Matthias Göggerle \\ Deutsches Museum / München \\ m.goeggerle@deutsches- \\ museum.de
}

\author{
Carola Dahlke \\ Deutsches Museum / München \\ C.dahlke@deutsches- \\ museum.de
}

\begin{abstract}
Being already an established method for nondestructive examination of cultural heritage objects from a conservational perspective, computed tomography is getting more and more popular for answering historical questions. As part of the three-year project $3 D$-Cipher, the technology will be applied to scan and digitize 61 historical cipher machines ranging from the late $19^{\text {th }}$ century to the 1990s. The German Federal Ministry of Education and Research funds the project in the eHeritage program ${ }^{1}$, which has the goal of supporting the digitalization of cultural heritage objects and making them accessible for researchers.

The aim of this contribution is to introduce the museum's collection as well as the project's idea and relevance to cryptologic researchers. Since 3D scans are able to provide non-destructive insights into our rare exhibits, we can thus hopefully contribute by making our devices available to scientists.
\end{abstract}

\section{Introduction}

The Deutsches Museum has a large collection of historical cipher machines ranging from the late 19 th century to the 1990s. As part of the threeyear 3D-Cipher project, $60+1^{2}$ objects of the cryptologic collection will be scanned and digitalized using computed tomography technology. The scan data and 3D-models will then be made available online in an open access format for international researchers.

The main goal of this project is to enable further research with the newly generated 3D-CT digitalization data of the cipher machines.

\footnotetext{
1 URL: https://www.geistes-und-sozialwissenschaftenbmbf.de/de/eHeritage-1736.html (26.05.2021)

2 The project includes a rare and well-preserved SG-41 device from WWII owned by a private collector.
}

Therefore, an adequate presentation of the project results is a necessity and an integral part of the project. The enrichment of the CT data with technical and scientific information is an important step in this process. To learn more about the requirements and wishes of the researchers, we want to use this contribution to present the project in its early stage to the crypto community and start a knowledge exchange.

\section{Computed tomography and other 3D scanning technologies}

\subsection{Surface scanning technologies}

As part of the digital surge of museum collections, 3D scanning techniques are increasingly being used in addition to $2 \mathrm{D}$ photographic recordings. The purpose and the outcome of 3D scans vary greatly with the different techniques applied. The most common are surface scanning technologies like photogrammetry, structured light scanning or laser scanning, which capture the surface of objects. ${ }^{3}$

\subsection{CT - functional principle}

The great advantage of the CT technology in comparison with the above-mentioned techniques is the possibility to scan the interior of objects. Using X-ray measuring from various angles, CT is not only able to show inside layers of objects, but can also be used to build digital 3D models. There are two main forms of CT technology, medical and industrial CT scanning. One of the main differences is the lower voltage in medical CT scans to minimize the radiation on the human body. Furthermore, the X-ray source will move around the body in medical CT scanners, whereas the object itself will usually move

\footnotetext{
${ }^{3}$ A good overview with advantages and disadvantages of the different technologies can be found here: URL: https://bitfab.io/blog/types-of-3d-scanning/ (26.05.2021).
} 
in the X-ray beam in industrial scanners. ${ }^{4}$ Industrial scanners also have a higher resolution, depending on the size of the scanner and of the object, ranging from five to $150 \mu \mathrm{m}$ Voxel size in Macro and Micro-CT scanners up to $0.5 \mu \mathrm{m}$ in Nano-CT scanners. The best resolution of medical scanners is around $70 \mu \mathrm{m}$ (Hanke, 2010; Du Plessis 2016).

All CT scanners use the penetration of the Xray beams to measure the density of different materials. Different materials absorb the radiation to different degrees; the picture on a detector panel appears darker or lighter depending on the absorption. The rotation of the source/ the object and the repeated measurements from different angles lead to a large number of $\mathrm{X}$-ray scans in the $\mathrm{x}-, \mathrm{y}$ - and $\mathrm{z}$-axis that can then be reconstructed to a 3D-CT-model (Luccichenti, 2005).

\subsection{D-Reconstruction}

Since the absorption of the X-rays is measured, the free space (i.e. air) is identified as a material with very low opacity. The result of the CT-scan is a digital cube, consisting of materials with different absorption rates. With the appropriate software, the parameters can be adjusted to see the different materials in a 3D reconstruction.

For further measurements or segmentation of different parts, e.g. rotors, gears, screws, the different materials, including the free spaces, have to be separated from each other. Automated processes exist, but for high quality results, elaborate manual editing is still required (Luccichenti, 2005).

To complicate things even more, visual artefacts appear in the scanning process where materials with high variance in density meet, i.e. metal artefacts e.g. beam hardening and scatter that result in black and white streaks (Boas, 2012).

\subsection{Usefulness of CT technology}

Despite these challenges, the possibility of nondestructive inspection of the interior of objects makes the CT technology an invaluable tool for researching unknown features of fragile objects.

\footnotetext{
${ }^{4}$ There are further subdivisions, which would go beyond the scope of this brief introduction. A good summary can be found in Hanke (2010).
}

These include changes or small fractures that otherwise would not be noticed.

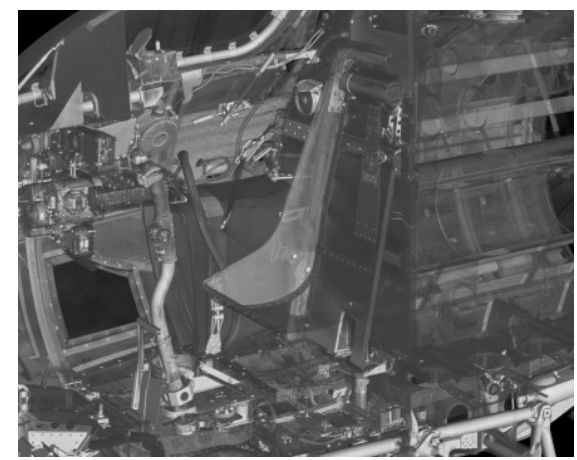

Fig. 1: 3D-CT-rendering of a WWII airplane cockpit (Fraunhofer IIS, EZRT/ Deutsches Museum, CC BY-SA 4.0). ${ }^{5}$

Depending on the quality of the scan data and the extent and accuracy of the segmentation process, even completely reverse engineered 3Dmodels are possible that include the details of mechanisms. ${ }^{6}$ These features are very useful concerning the cryptologic collection researched in this project.

\section{Collection overview}

The museum's collection of cipher machines is very extensive, and depicts the variance of 120 years of Central European mechanical cryptology. The collection can be categorized into five chronological periods:

\subsection{The beginning of mechanical encryption around 1900}

The oldest cipher machines in the collection date from before 1900 and do not yet contain any complicated techniques. They are particularly interesting because some of them are completely unknown; e.g. a very early prototype of a cipher machine was donated to the museum by the Danish inventor Alexis Køhl himself. Other machines from the 1900 s to the 1920 s follow, e.g. devices from Friedrich Rehmann and

\footnotetext{
${ }^{5}$ Similar quality can be expected for the cipher devices. Link to the project, URL: https://www.deutschesmuseum.de/presse/presse-2019/me-163/\#c137993

(26.05.2021)

${ }^{6}$ The team from Prof. Philip Withers at the University of Manchester scanned and segmented an Enigma in 2018. URL: https://www.manchester.ac.uk/discover/news/x-rayimaging-reveals-the-secrets-inside-the-enigma-machine/ (26.05.2021).
} 
Alexander von Kryha. CT scans of these rare devices will complete the existing data available so far.

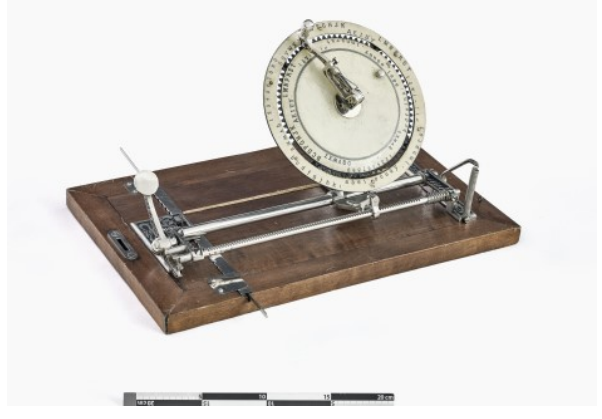

Fig. 2: Index Typewriter „Diskret” by Friedrich Rehmann, (Deutsches Museum/ Konrad Rainer, Inv.-No. 67624, CC BY-SA 4.0).

\subsection{Cipher machines of World War II}

The collection contains mainly German cipher machines from Heimsoeth \& Rinke, Siemens, Lorenz and Wanderer Werke AG, but also includes a variety of Hagelin machines from A. B. Cryptoteknik and L. C. Smith. Some types are already very well analyzed (i.e. army and naval Enigma models). Others, e.g. the German Siemens secret teleprinter T52, are much rarer and their interesting history is less present. For this very reason, scan data give the opportunity for the not-so-well-known devices to be examined more closely.

\subsection{Cipher machines of the post-war period}

Shortly after the end of the Second World War, a large number of different cipher machines were developed. Transmission was still mainly by radio or telegraph cable. Many of these devices are still not well known.

This period is represented in the collection by some successors to Enigma models, such as $\mathrm{NeMa}$ and Fialka, and Hagelin devices from Crypto AG and Rudolph Hell. Other items of a different kind by the Swedish company Transvertex and the company Stenographic Machines, Inc. add to the picture.

So-called mixers, i.e. devices meant to encrypt with random sequences on punched tape, are represented by devices of the companies CryptoAG and Siemens \& Halske.

In the context of the gentlemen's agreement between Boris Hagelin and William Friedman (see e.g. the declassified report of W. Friedman, 1955), the comparison of scan data from various Hagelin devices of the C- and CX-series in the collection is planned. Particular interest lies in a possible detection of differences in the mechanics that are usually not accessible without opening and thus destroying the rare devices.

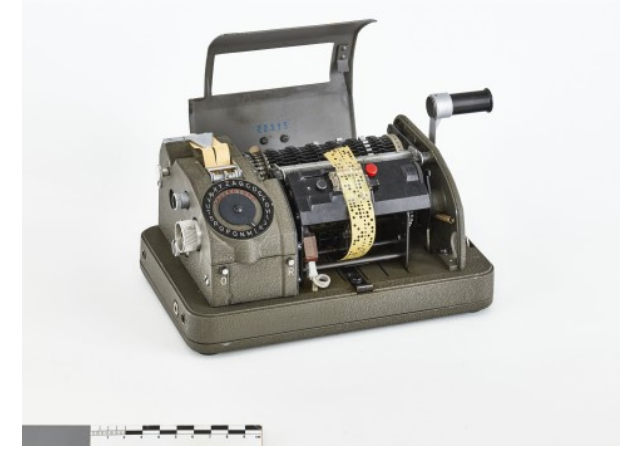

Fig. 3: Hagelin CX-52/RT (Deutsches Museum/ Konrad Rainer, Inv.-No. 2017-389, CC BY-SA 4.0).

\subsection{Beginning of the computer age from 1965}

From 1965, encryption algorithms are no longer mechanically driven, but executed on circuit boards. Almost none of the devices from this period have been investigated yet, and for a comprehensive study of the algorithms, contemporary documents are required.

The collection is rather extensive in this section and contains devices of the companies Mils Electronic, Crypto AG, Tele-Security Timmann, Telta. Telefunken, Philips and ANT Nachrichtentechnik GmbH.

Since the electronic components from this period are still easily recognizable, the CT scans will enable to clarify how specific functions and algorithms have been implemented electronically.

\subsection{Computer age from 1980}

From the 1980s onwards, almost all devices are completely unknown, and had been kept top secret until a few years ago. Most are grey or black tightly welded boxes and from the outside it is difficult to see what might be inside or what they were used for. Above all, documentation is hard to come by. 


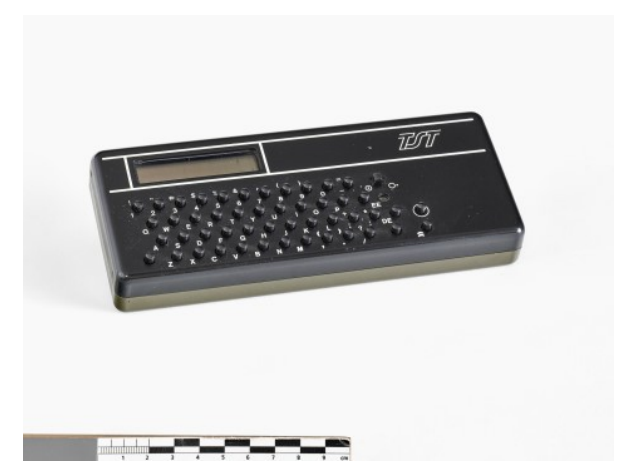

Fig. 4: TST 3226 by Tele Security Timmann (Deutsches Museum/ Konrad Rainer, Inv.-No. 2017-410, CC BY-SA 4.0).

The devices were mostly designed for data and voice encryption and transmitted via telephone. Random number generators, modems and faxes are part of the exhibits from this period. CT scans are essential to uncover this part of our collection. This way, one can at least narrow down what the device did. Apart from studying the components inside and identifying their modes of operation, we are in particular interested in revealing the object histories of some devices. Tele-Security Timmann devices from this period are said to contain a special copy protection, i.e. a substance that fills the device and destroys the inner parts if it is opened by force. We are curious to see whether CT scans will reveal any information.

\section{Conclusion}

\subsection{CT \& the collection}

The CT technology has the possible features to answer open research issues across the spectrum of the collection. At least two WWII machines will be processed via segmentation of important functional parts to enable a direct comparison between them. From every other object, we intend to create a 3D-CT-model that can be used for further editing in the future by international researchers. The Open Access Policy is crucial at this point and we hope to engage in collaboration with various experts as part of the project.

\subsection{Researching the data}

The CT data can be analyzed with proprietary software. Although the operation of the software requires a certain knowledge and the handling of the data makes a high-end computer, e.g. with lots of RAM, a necessity, we will freely share the data with international researchers for further enquiry.

\subsection{Presentation of the data}

The above-mentioned process is important for research purposes, but is hidden from a broader audience due to the technological requirements. Therefore, we plan to show the 3D-CT models in an online web viewer with the possibility to download these models for further use. ${ }^{7}$ This two-way approach with research data and online exhibition will hopefully path a way to uncover the last secrets of the cipher machines.

\subsection{Upcoming roadmap}

The first images and 3D reconstructions are expected in the summer 2021. Following this, we will enrich and research the CT data in the museum and prepare the publications. We aim to submit a long paper in the following HistoCrypt proceedings, including first research results.

\section{References}

Anton Du Plessis et al, 2016: Laboratory X-ray micro-computed tomography: a user guideline for biological samples. In GigaScience 2017, 6(6), p. $1-11$.

Franz Edward Boas and Dominik Fleischmann: CT artifacts: Causes and reduction techniques. In Imaging in Medicine 2012, 4 (2), p. 229-240.

Giacomo Luccichenti et al.: 3D reconstruction techniques made easy: Know-how and pictures. In European Radiology 2005, 15 (10), p. 2146-2156.

Randolph Hanke: Computertomographie in der Materialprüfung. Stand der Technik und aktuelle Entwicklungen. Fürth 2010.

William Friedman, 1955, National Security Archive REF: A2436259. Report of Visit to Crypto A.G. (Hagelin) by William F. Friedman, Special Assistant to the Director, National Security Agency. March 15, 1955. Top Secret.

Webpages

https://bitfab.io/blog/types-of-3d-scanning/ (26.05.2021).

https://www.deutsches-museum.de/presse/presse2019/me-163/\#c137993 (26.05.2021).

https://www.geistes-und-sozialwissenschaftenbmbf.de/de/eHeritage-1736.html (26.05.2021).

https://www.manchester.ac.uk/discover/news/x-rayimaging-reveals-the-secrets-inside-the-enigmamachine/ (26.05.2021).

https://musices.gnm.de/ (26.05.2021)

7 A possible solution was used by the Germanisches National Museum in cooperation with the Fraunhofer EZRT in a project that $\mathrm{CT}$ scanned historical musical instruments. URL: https://musices.gnm.de/ (26.05.2021). 